\title{
Retrospective clinicopathological study of 418 odontogenic cysts
}

\author{
Sergio Núñez-Urrutia ${ }^{1}$, Rui Figueiredo ${ }^{2}$, Cosme Gay-Escoda ${ }^{3}$
}

${ }^{1}$ DDS. Master degree program in Oral Surgery and Implantology. Barcelona University Dental School

${ }^{2}$ DDS. Master degree program in Oral Surgery and Implantology. Associate professor of Oral Surgery and Professor of the Master degree program of Oral Surgery and Implantology. University of Barcelona Dental School. Researcher of the IDIBELL institute

${ }^{3}$ MD, DDS, PhD. Chairman of Oral and Maxillofacial Surgery. Director of the Master degree program of Oral Surgery and Implantology. University of Barcelona Dental School. Coordinating researcher of the IDIBELL Institute. Maxillofacial Surgeon, Teknon Medical Center. Barcelona, Spain

Correspondence:

Centro Médico Teknon

Instituto de Investigación IDIBELL

Vilana 12

08022 - Barcelona (Spain)

cgay@ub.edu

Received: 13/04/2009

Accepted: $21 / 02 / 2010$

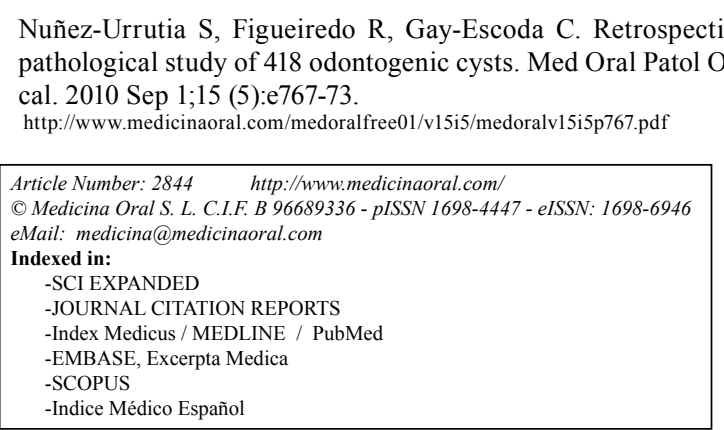

\begin{abstract}
Objectives: To determine the relative incidence of odontogenic cysts and to identify the main clinicopathological features among patients treated in the Oral Surgery Department of the Dental Clinic of the University of Barcelona (Spain).

Study design: A retrospective observational study was made of 418 odontogenic cysts diagnosed in 380 patients included in the database of 1235 histopathological diagnoses. The subjects were treated in the Master degree program of Oral Surgery and Implantology of the University of Barcelona in the period 1997-2006. The following variables were recorded: gender, age, clinical characteristics of the lesions (size and location), radiological features, duration, treatment, complications and relapses. A descriptive analysis was made of the study variables, using the SPSS version 15.0.

Results: The incidence of odontogenic cysts was 33.8\%. The mean patient age at appearance of the lesion was 42 years (range 7-83). The cysts were slightly more prevalent in males (58.4\%). The lesion size ranged from 2-60 mm, with a mean size of $18.4 \mathrm{~mm}$. The most frequent diagnosis was radicular cyst $(50.2 \%)$. The most common location of the odontogenic cysts was in the mandible (61.5\%), particularly the lower third molar region (36.8\%).

Conclusions: The most frequently diagnosed lesion was the radicular cyst. Odontogenic cysts were seen to be slightly more prevalent in males, and showed a high mandibular incidence. Knowledge of the biological and histological behavior of odontogenic cysts and their frequency are key aspects for ensuring early detection and adequate treatment.
\end{abstract}

Key words: Cysts, odontogenic cysts, maxillary cysts, malignization. 


\section{Introduction}

A cyst is defined as a cavity with liquid, semiliquid or gaseous contents, enveloped by an epithelial tissue membrane (1-3). Odontogenic cysts (OCs) are the most frequent maxillary lesions and present a membrane composed of epithelial tissue of odontogenic origin. The etiology of OCs is explained by the presence of odontogenic cell remains entrapped within the bone tissue or gingival tissue covering the maxilla or mandible, such as for example the epithelial remains of Malassez, the dental lamina or the enamel organ (developmental OCs). Nevertheless, in other cases, the cysts are produced by inflammatory processes (inflammatory OCs) (Table 1). In most cases the lesions are true cysts, with a complete and sealed epithelial lining (1-3). The most recent classification of the World Health Organization (WHO) reallocated keratocyst (keratinized primordial cyst) within the classification of maxillary tumors under the term "keratocystic odontogenic tumor" (KOT) (4). Its slow, expansive and non-infiltrating growth pattern constitutes clear evidence of its benign nature - a situ- ation which may facilitate late diagnosis. The appearance of signs such as cortical expansion (Fig. 1), dental displacement or the appearance of pain or infectious symptoms should alert the clinician to the possible presence of cyst pathology. Routine radiographic evaluation based on panoramic radiographies reveals the presence of characteristic radiotransparent lesions (Fig. 2) (1-3, 5).

The treatment of choice is conditioned by a number of factors such as the size of the lesion, its location, the possible involvement of neighboring anatomical structures, or damage to dental structures, among others $(1-3,5)$. The diagnosis of OC is established from the different clinical and radiological features, though the final diagnosis is based on histopathological study of the lesion $(1-3,5,6)$.

The present study was designed in view of the high prevalence of OCs and the need to know their clinicopathological characteristics, with the aim of developing specific protocols for ensuring an early diagnosis and prompt and adequate treatment.

Table 1. World Health Organization classification of odontogenic cysts (2005).

\begin{tabular}{|c|c|c|}
\hline \multicolumn{2}{|c|}{ EPITHELIAL CYSTS } & NON-EPITHELIAL \\
CYSTS
\end{tabular}




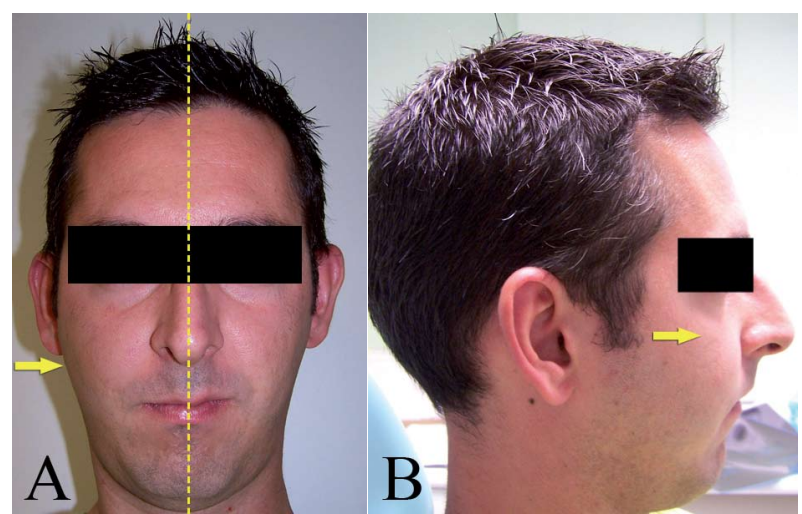

Fig. 1. Upper maxillary cyst. (A) Facial asymmetry produced by vestibular cortical expansion due to the presence of an odontogenic cyst in the right posterior zone of the upper maxilla. (B) Detail of the nasogenian sulcus.

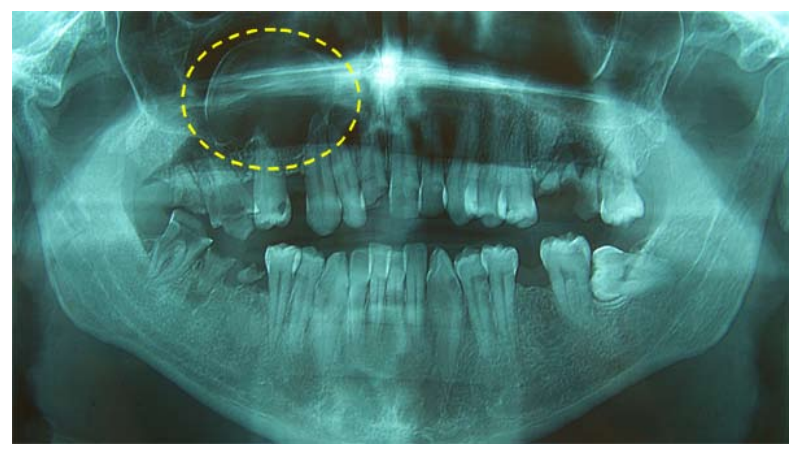

Fig. 2. Residual cyst in the first quadrant, displacing the sinus membrane.

\section{Patients and Methods}

A retrospective study was made of 1235 anatomopathological diagnosis of peri- and intraoral lesions established in the period 1997-2006, in the Master degree program of Oral Surgery and Implantology of the University of Barcelona (Spain), with the purpose of identifying patients with odontogenic cyst (OC). The study data were collected by a single observer. The samples obtained were fixed in $10 \%$ formaldehyde and sent to the Pathology Service (Bellvitge University Hospital, Barcelona, Spain) for processing and staining with hematoxylin-eosin. The samples were examined under the light microscope and a histological diagnosis was made.

The inclusion criteria involved histological confirmation of OC. Lesions with histological findings not compatible with OC were excluded from the study. The following variables were recorded: gender, age, radiological features of the lesions, location and size (measuring the greater diameter of the lesion on the panoramic radiographies), duration, treatment provided, histological findings and possible relapses. In all cases radiological study was based on the panoramic radiography, though in some cases periapical or occlusal radiographies were also requested, as well as computed tomography (CT) scans, to complement the imaging diagnostic data. The follow-up period was evaluated in months, and the perand postoperative complications associated to each OC were registered. The data obtained were subjected to descriptive statistical analysis using the SPSS version 15.0 statistical package (SPSS Inc., Chicago, USA) (license of the University of Barcelona, Spain).

\section{Results}

A total of 418 OCs were registered (in 380 patients), out of a total of 1235 biopsies performed in our Service corresponding to $33.8 \%$ of all the lesions referred for histopathological study during the examined time period (1997-2006). Seven OC variants were identified.

The most commonly diagnosed OC was the radicular cyst $(\mathrm{RC})$, representing $50.2 \%$ of the total $(n=210)$. The most common OC location was the mandible $(n=257$; $61.5 \%$ ), where in turn the third quadrant was the most frequently affected zone $(n=138 ; 33 \%)$. (Table 2$)$ shows the frequency of appearance of OCs diagnosed in both maxillas. The teeth most often associated with the presence of OCs were the lower third molars (36.8\%), followed by the mandibular first and second molars (13\%) (Table 3).

Table 2. Frequency of the different types of odontogenic cysts.

\begin{tabular}{|l|c|c|c|}
\hline \multicolumn{1}{|c|}{ Odontogenic cysts } & $\begin{array}{c}\text { Maxilla } \\
(\mathbf{n} ; \text { relative \%) }\end{array}$ & $\begin{array}{c}\text { Mandible } \\
(\mathbf{n} ; \text { relative \%) }\end{array}$ & $\begin{array}{c}\text { Total cases } \\
(\mathbf{n} ; \text { relative \%) }\end{array}$ \\
\hline Radicular cyst & $(\mathrm{n}=118 ; 56.2 \%)$ & $(\mathrm{n}=92 ; 43.8 \%)$ & $(\mathrm{n}=210 ; 50.2 \%)$ \\
\hline Follicular cyst & $(\mathrm{n}=28 ; 30.8 \%)$ & $(\mathrm{n}=63 ; 69.2 \%)$ & $(\mathrm{n}=91 ; 21.8 \%)$ \\
\hline Paradental cyst & $(\mathrm{n}=2 ; 2.3 \%)$ & $(\mathrm{n}=85 ; 97.7 \%)$ & $(\mathrm{n}=87 ; 20.8 \%)$ \\
\hline Residual cyst & $(\mathrm{n}=9 ; 50 \%)$ & $(\mathrm{n}=9 ; 50 \%)$ & $(\mathrm{n}=18 ; 4.3 \%)$ \\
\hline Periodontal lateral cyst & $(\mathrm{n}=3 ; 42.8 \%)$ & $(\mathrm{n}=4 ; 57.2 \%)$ & $(\mathrm{n}=7 ; 1.7 \%)$ \\
\hline Primordial cyst & 0 & $(\mathrm{n}=4 ; 100 \%)$ & $(\mathrm{n}=4 ; 1 \%)$ \\
\hline Adult gingival cyst & $(\mathrm{n}=1 ; 100 \%)$ & 0 & $(\mathrm{n}=1 ; 0.2 \%)$ \\
\hline TOTAL & $(\mathrm{n}=\mathbf{1 6 1 ;} \mathbf{3 8 . 5 \% )}$ & $(\mathrm{n}=\mathbf{2 5 7} ; \mathbf{6 1 . 5 \% )}$ & $\mathbf{( n = 4 1 8 ; \mathbf { 1 0 0 } \% )}$ \\
\hline
\end{tabular}


Table 3. Frequency of the different types of odontogenic cysts by dental groups.

\begin{tabular}{|c|c|c|c|c|c|}
\hline Upper maxilla & Cases & Relative \% & Mandible & Cases & Relative \% \\
\hline Central incisors & 35 & $8.4 \%$ & Central incisors & 10 & $2.4 \%$ \\
\hline Lateral incisors & 33 & $7.9 \%$ & Lateral incisors & 6 & $1.4 \%$ \\
\hline Canines & 30 & $7.2 \%$ & Canines & 7 & $1.7 \%$ \\
\hline Premolars & 23 & $5.5 \%$ & Premolars & 26 & $6.2 \%$ \\
\hline $1^{\text {st }}$ and $2^{\text {nd }}$ molars & 31 & $7.4 \%$ & $1^{\text {st }}$ and $2^{\text {nd }}$ molars & 54 & $13 \%$ \\
\hline $3^{\text {rd }}$ molars & 9 & $2.1 \%$ & $3^{\text {rd }}$ molars & 154 & $36.8 \%$ \\
\hline TOTAL & $\mathbf{1 6 1}$ & $\mathbf{3 8 . 5 \%}$ & TOTAL & $\mathbf{2 5 7}$ & $\mathbf{6 1 . 5 \%}$ \\
\hline
\end{tabular}

Table 4. Relationship between treatment and intra- and postoperative complications.

\begin{tabular}{|c|c|c|c|c|c|c|c|}
\hline Treatment & Infection & $\begin{array}{c}\text { Nerve } \\
\text { damage }\end{array}$ & $\begin{array}{l}\text { Sinus/nasal } \\
\text { mucosa } \\
\text { perforation }\end{array}$ & Sinusitis & $\begin{array}{c}\text { Pulp } \\
\text { necrosis }\end{array}$ & $\begin{array}{c}\text { Alveolar } \\
\text { osteitis }\end{array}$ & Total \\
\hline $\begin{array}{c}\text { Removal with or without } \\
\text { extraction } \\
(\mathrm{n}=300 ; 72 \%)\end{array}$ & 0 & $(\mathrm{n}=11 ; 3.6 \%)$ & $(n=6 ; 2 \%)$ & 0 & $(\mathrm{n}=1 ; 0.3 \%)$ & $(\mathrm{n}=1 ; 0.3 \%)$ & \\
\hline $\begin{array}{c}\text { Cyst removal + apicoectomy } \\
+ \text { retrograde filling } \\
(\mathrm{n}=109 ; 26 \%)\end{array}$ & $(\mathrm{n}=6 ; 5.5 \%)$ & $(\mathrm{n}=1 ; 1 \%)$ & $(\mathrm{n}=2 ; 1.7 \%)$ & $(\mathrm{n}=1 ; 1 \%)$ & 0 & 0 & \\
\hline $\begin{array}{c}\text { Cyst decompression } \\
(\mathrm{n}=8 ; 2 \%)\end{array}$ & 0 & 0 & $(\mathrm{n}=4 ; 50 \%)$ & 0 & 0 & 0 & \\
\hline $\begin{array}{l}\text { TOTAL } \\
(n=418)\end{array}$ & $(\mathrm{n}=6 ; 1.4 \%)$ & $(\mathrm{n}=12 ; 2.9 \%)$ & $(\mathrm{n}=12 ; 2.9 \%)$ & $(\mathrm{n}=1 ; 0.2 \%)$ & $\begin{array}{r}(\mathrm{n}=1 \\
0.2 \%)\end{array}$ & $(\mathrm{n}=1 ; 0.2 \%)$ & \\
\hline
\end{tabular}

Table 5. Comparison of the incidence of the different types of odontogenic cysts with some of the most important published series.

\begin{tabular}{|c|c|c|c|c|}
\hline Odontogenic cysts & $\begin{array}{c}\text { Ledesma et al. } \\
\mathbf{( 2 0 0 0 ) ( 1 )} \\
\mathbf{3 0 4} \text { cases }\end{array}$ & $\begin{array}{c}\text { Mosqueda-Taylor et al. } \\
\mathbf{( 2 0 0 2 ) ( 2 )} \\
\mathbf{8 5 6} \text { cases }\end{array}$ & $\begin{array}{c}\text { Ochsenius et al. } \\
\text { (2007)(3) } \\
\mathbf{2 9 4 4} \text { cases }\end{array}$ & $\begin{array}{c}\text { Núñez-Urrutia et al. } \\
\text { (2010) } \\
\mathbf{4 1 8} \text { cases }\end{array}$ \\
\hline Radicular cyst & $38.8 \%$ & $39.9 \%$ & $50.7 \%$ & $50.2 \%$ \\
\hline Follicular cyst & $35.5 \%$ & $33 \%$ & $18.5 \%$ & $21.8 \%$ \\
\hline Paradental cyst & & $1.4 \%$ & $3.8 \%$ & $20.8 \%$ \\
\hline Residual cyst & $4.9 \%$ & $2.2 \%$ & $11.2 \%$ & $4.3 \%$ \\
\hline Periodontal lateral cyst & $1 \%$ & $0.8 \%$ & $0.6 \%$ & $1.7 \%$ \\
\hline Primordial cyst & $18.7 \%$ & $21.5 \%$ & $0.3 \%$ & $1 \%$ \\
\hline Adult gingival cyst & & & & $0.2 \%$ \\
\hline
\end{tabular}


The gender distribution showed a slight male predilection $(n=244 ; 58.4 \%)$, corresponding to a ratio of $1.4 / 1$. The mean patient age at the time of presentation of the lesions was 42 years (standard deviation (SD) 15), with a range of 7-83 years. OCs appeared most frequently between the third and fourth decades of life.

A panoramic radiographic study was requested in all patients. In some cases, additional radiological studies were requested: computed tomography (CT) $(n=90$; $24 \%)$, occlusal radiographies $(n=13 ; 3 \%)$, lateral projections $(n=9 ; 2 \%)$, and Waters X-rays $(n=4 ; 1 \%)$, with the purpose of determining the size of the lesion and evaluating the possible involvement of adjacent structures. The lesion extension ranged from 2-60 mm, with a mean size of $18.4 \mathrm{~mm}$.

In relation to surgical treatment, cystic enucleation procedures with or without tooth removal predominated $(n=300 ; 72 \%)$, followed by periapical surgery (lesion resection plus apicoectomy and retrograde filling) $(\mathrm{n}=109 ; 26 \%)$. (Table 4$)$ shows the relationship between the treatments provided and the complications observed during and after surgery. Antral perforations were intraoperatively repaired by placing a reabsorbable collagen membrane (Bio-Guide; Geistlich Pharma, Wolhusen, Switzerland). As regards sensory alterations secondary to inferior alveolar nerve damage $(n=12 ; 2.9 \%)$, total symptoms remission was recorded in $83 \%$ of the cases. Nevertheless, in two patients a slight hypoesthesia was still present in the last follow-up examination. All other complications were satisfactorily resolved. The mean duration of follow-up was 11.7 months (range 1-72). In $14.8 \%$ of the cases $(n=62)$ the surgically treated lesions required additional surgery, including surgical curettage, periapical surgery or dental extraction, among other procedures.

\section{Discussion}

\section{Clinicopathological features}

When comparing the results obtained with those published by other authors, a number of differences have been noted (Table 5). In our series, the most commonly diagnosed odontogenic cyst (OC) was the radicular cyst (RC) $(\mathrm{n}=210 ; 50.2 \%)$ - an observation that coincides with the literature (1-3). The concordance between our own findings and the different published articles regarding RC distribution by dental arch and dental group may be explained by the high prevalence of traumatisms and conservative treatments carried out for esthetic reasons in this group of teeth (6). The consulted literature showed no significant differences in distribution by patient age or gender, despite the variability in sample sizes $(1,3,6)$.

The second most frequently diagnosed lesion was follicular cyst (FC), representing $21.8 \%$ of the total $(n=91)$, in coincidence with the figures found in the literature (1-3). FC is associated to the crown of permanent teeth and impacted odontomas, and in a lesser proportion of cases it can affect impacted deciduous teeth (1-3, 7,8). A follicular space of over $3 \mathrm{~mm}$ should be clinically regarded as a dentigerous or follicular cyst, since histologically epithelial thickening is usually noted as a result of the inflammatory changes induced by the accumulation of fluid between the crown and the dental follicle (9). Curran et al. (7) observed a $45-75 \%$ frequency of FCs, though most consulted studies report a considerably lower percentage (18.5\%-35.5\%) (1-3). FCs have a strong predilection for the mandible, as was confirmed in our study (69.2\% of all cases) (1-3). Although usually unilateral, there are multiple reports of bilateral FCs in nonsyndromic patients $(7,8)$. Due to the slow and asymptomatic growth that characterizes FCs, these lesions can reach large sizes (6). The extraction of impacted teeth not amenable to repositioning within the dental arch, or the periodic follow-up of those teeth where extraction is not clearly indicated, are the measures that help avoid possible complications of FCs (6-8).

Paradental cyst (PC) is of inflammatory origin and mainly affects semi-impacted lower third molars (submucosal impaction), though there have also been reports of cases associated with the first molar and lower second premolar $(10,11)$. These lesions tend to manifest as characteristic "croissant" shaped radiotransparencies. PCs are generally found in patients in the third decade of life, though pediatric cases have also been documented $(10,11)$. These cysts develop near the cervical margin of the lateral portion of the root as a consequence of periodontal pocket inflammation. Most authors agree that pericoronitis or chronic inflammation play a role in the etiopathogenesis of PC $(10,11)$. In our series PC was the third most common type of OC $(20.8 \%$ of the total $)$ $(n=87)$. This is in contradiction to the results reported by both Mosqueda-Taylor et al. (2), with a frequency of $1.4 \%$ for PCs, and Ochsenius et al. (3), with a proportion of $3.8 \%$ (Table 5 ). These differences may be explained by the origin of our patient series - ours being an Oral Surgery Unit, where most patients are seen for problems related to impacted third molars.

In turn, primordial cysts (PMCs) represented $1 \%$ of all the OCs diagnosed and treated in our Service. This percentage is significantly lower than in most other studies (1-3). The documented cases show a slight female predilection, in contrast to the observations of other investigators (1-3). During the elaboration of the present study we included "keratocystic odontogenic tumor" (KOT)(previously referred to as keratocyst (KC)) in the series of PMCs, to be able to compare our results with published studies, since most authors in the consulted literature tended to unite both concepts (PMC and $\mathrm{KC}$ ) (1-3). The growth potential of KOT is influenced by its active epithelial proliferation. Some authors point to a relationship between cytokeratin-10 expression and the 
high recurrence rate seen for some KOTs (12). Ali (13) studied the tissues obtained with immunohistochemical techniques, using anti-EMMPRIN (extracellular matrix metalloproteinase inducer) antibodies. This author reported different results among OCs according to their expression of EMMPRIN - significantly increased aggressivity and recurrence being noted for those lesions with increased EMMPRIN expression. These studies showed KOT to be the OC with the most intense EMMPRIN expression.

In turn, the fourth most common cyst in our series was residual cyst $(\mathrm{RC})(\mathrm{n}=18 ; 4.3 \%)$. $\mathrm{RC}$ is an inflammatory $\mathrm{OC}$ resulting from incomplete resection of cystic tissue in the periapical zone of a previously extracted pathological tooth. The reviewed literature indicates that RCs are most frequently observed between the third and fourth decades of life in coincidence with our own findings (1-3). In the present series no predilection for either the upper maxilla or the mandible was observed $(50 \%)$. Lateral periodontal cyst (LPC) is a developmental cyst with a male predilection that usually appears in adults between the fourth and sixth decades of life $(1-3,14,15)$. LPC manifests as a rounded, "inverted pear" shaped and well delimited radiotransparent image that is often mistaken for an OC of inflammatory origin. The preserved vitality of the affected teeth offers the key to differential diagnosis in this case. In our retrospective series LPCs were more frequent $(\mathrm{n}=7 ; 1.7 \%)$ than in the consulted literature (0.8-0.9\%) (1-3, 14,15). Rasmusson et al. (14), in an analysis of 32 LPCs, found the most frequent location to be between the canines and premolars, with a clear mandibular predilection. Our own experience with these lesions comprised three cases in the upper maxilla located between the lateral incisors (43\%), and four in the mandible (57\%) - this distribution coinciding with the reports in the literature $(14,15)$.

\section{Considerations regarding treatment}

Pulpectomy is the treatment of choice in the case of a radiotransparent lesion measuring under $10-15 \mathrm{~mm}$ in diameter and associated to a necrotic tooth $(6,16)$. In the presence of signs and/or symptoms, or an increase or persistence of the radiotransparent image 6 months after root canal treatment, the recommended procedure is periapical surgery of the tooth, with complete removal of the cyst, apicoectomy and retrograde filling $(6,16)$.

In our Oral Surgery service, a total of 109 surgical procedures (periapical surgery) were made $(26 \%)$, with a failure rate of 5.5\%. Carrillo et al. (16) evaluated 70 periapical lesions subjected to surgical treatment, with radiological follow-up of 12 months. They recorded a significantly poorer prognosis in the case of large lesions, in coincidence with our own observations. A histopathological study was made of all the OCs, with a view to securing a firm diagnosis, since the literature describes cases with clinical and radiological character- istics suggestive of benignity, which posteriorly proved to be malignant $(1,5,16,17)$.

In lesions over $25-30 \mathrm{~mm}$ in size, panoramic radiographies are unable to produce sufficient information to plan an adequate surgical treatment (5, 7-9). In addition, to panoramic X-rays, in our series we requested computed tomography scans, occlusal and lateral cranial radiographies and the Waters projection to more precisely establish the extent of the lesions and minimize the risk of intraoperative complications with any OC affecting other neighboring anatomical structures. In the case of lesions over $30 \mathrm{~mm}$ in size, with the displacement of adjacent structures or with buccal/lingual bone plate perforation (7-9), surgical decompression with or without extraction of the causal tooth is recommended. Surgical decompression was described by Brondum et al. (12) as an alternative to the enucleation of large cysts, without the need for extensive mandibular resections. Some authors such as August et al. (18) consider surgical decompression to favor a change in the histological architecture of KOTs, lessening their tendency towards recurrence.

The decrease in intracyst pressure achieved with surgical decompression allows a reduction in lesion volume and favors bone growth $(12,18,19)$. This technique also affords information on the histological type of OC involved $(12,18,19)$. Surgical decompression moreover considerably reduces complications such as mandibular fracture, oroantral fistulas or damage to nerve structures $(12,18,19)$. However, decompression has two important disadvantages. On one hand, the patient must cooperate to ensure good buccodental hygiene, and must report to the necessary follow-up visits. On the other hand, the sample obtained for biopsy purposes only represents part of the cyst, and a firm histopathological diagnosis of the complete lesion may therefore not be obtained. There have been reports of KOTs lacking keratin in certain zones of the cyst wall (partially keratinized cysts) (20). Correct histological diagnosis following resection of the entire lesion can exceptionally identify carcinomatous cells within a well differentiated epithelium. This in turn radically changes the treatment recommendations, due to the possible presence of a primary intrabony carcinoma (17).

\section{Conclusions}

The present study confirms the predominance of radicular cysts $(50.2 \%)$, followed by follicular cysts $(21.8 \%)$. Odontogenic cysts were seen to be slightly more prevalent in males (58.4\%), and showed a high mandibular incidence $(61.5 \%)$. Keratocysts were infrequent $(1 \%)$, in contrast to the increased presence of paradental cysts $(20 ., 8 \%)$. Knowledge of the biological and histological behavior of odontogenic cysts and their frequency are key aspects for ensuring early detection and adequate treatment. 


\section{References}

1. Ledesma-Montes C, Hernández-Guerrero JC, Garcés-Ortíz M. Clinico-pathologic study of odontogenic cysts in a Mexican sample population. Arch Med Res. 2000;31:373-6.

2. Mosqueda-Taylor A, Irigoyen-Camacho ME, Diaz-Franco MA, Torres-Tejero MA. Odontogenic cysts. Analysis of 856 cases. Med Oral. 2002;7:89-96.

3. Ochsenius G, Escobar E, Godoy L, Peñafiel C. Odontogenic cysts: analysis of 2,944 cases in Chile. Med Oral Patol Oral Cir Bucal. 2007;12:E85-91.

4. Madras J, Lapointe H. Keratocystic odontogenic tumour: reclassification of the odontogenic keratocyst from cyst to tumour. J Can Dent Assoc. 2008;74:165-165h.

5. Nary Filho H, Matsumoto MA, Fraga SC, Gonçales ES, Sérvulo F. Periapical radiolucency mimicking an odontogenic cyst. Int Endod J. 2004;37:337-44.

6. Garcia-Pola MJ, Gonzalez M, Lopez JS. Odontogenic maxillary cyst. Clinicopathologic. diagnostic and therapeutic considerations. Med Oral. 1997;2:219-241.

7. Curran AE, Damm DD, Drummond JF. Pathologically significant pericoronal lesions in adults: Histopathologic evaluation. J Oral Maxillofac Surg. 2002;60:613-7.

8. Iatrou I, Theologie-Lygidakis N, Leventis M. Intraosseous cystic lesions of the jaws in children: a retrospective analysis of 47 consecutive cases. Oral Surg Oral Med Oral Pathol Oral Radiol Endod. 2009;107:485-92.

9. Edamatsu M, Kumamoto H, Ooya K, Echigo S. Apoptosis-related factors in the epithelial components of dental follicles and dentigerous cysts associated with impacted third molars of the mandible. Oral Surg Oral Med Oral Pathol Oral Radiol Endod. 2005;99:17-23. 10. Philipsen HP, Reichart PA, Ogawa I, Suei Y, Takata T. The inflammatory paradental cyst: a critical review of 342 cases from a literature survey, including 17 new cases from the author's files. J Oral Pathol Med. 2004;33:147-55.

11. Morimoto Y, Tanaka T, Nishida I, Kito S, Hirashima S, Okabe S, et al. Inflammatory paradental cyst (IPC) in the mandibular premolar region in children. Oral Surg Oral Med Oral Pathol Oral Radiol Endod. 2004;97:286-93.

12. Brøndum N, Jensen VJ. Recurrence of keratocysts and decompression treatment. A long-term follow-up of forty-four cases. Oral Surg Oral Med Oral Pathol. 1991;72:265-9.

13. Ali MA. Expression of extracellular matrix metalloproteinase inducer in odontogenic cysts. Oral Surg Oral Med Oral Pathol Oral Radiol Endod. 2008;106:258-63.

14. Rasmusson LG, Magnusson BC, Borrman H. The lateral periodontal cyst. A histopathological and radiographic study of 32 cases. Br J Oral Maxillofac Surg. 1991;29:54-7.

15. Formoso Senande MF, Figueiredo R, Berini Aytés L, Gay Escoda C. Lateral periodontal cysts: a retrospective study of 11 cases. Med Oral Patol Oral Cir Bucal. 2008;13:E313-7.

16. Carrillo C, Peñarrocha M, Bagán JV, Vera F. Relationship between histological diagnosis and evolution of 70 periapical lesions at 12 months, treated by periapical surgery. J Oral Maxillofac Surg. 2008;66:1606-9.

17. Chaisuparat R, Coletti D, Kolokythas A, Ord RA, Nikitakis NG. Primary intraosseous odontogenic carcinoma arising in an odontogenic cyst or de novo: a clinicopathologic study of six new cases. Oral Surg Oral Med Oral Pathol Oral Radiol Endod. 2006;101:194200.

18. August M, Faquin WC, Troulis MJ, Kaban LB. Dedifferentiation of odontogenic keratocyst epithelium after cyst decompression. J Oral Maxillofac Surg. 2003;61:678-83.

19. Jung YS, Lee SH, Park HS. Decompression of large odontogenic keratocysts of the mandible. J Oral Maxillofac Surg. 2005;63:26771.

20. Kolokythas A, Fernandes RP, Pazoki A, Ord RA. Odontogenic keratocyst: to decompress or not to decompress? A comparative study of decompression and enucleation versus resection/peripheral ostectomy. J Oral Maxillofac Surg. 2007;65:640-4.

\section{Acknowledgements}

This study has been carried out by the research group in "Dental and Maxillofacial Pathology and Treatment" of the Institut d'Investigació Biomèdica de Bellvitge (IDIBELL), with financial support from the oral surgery teaching-healthcare agreement among the University of Barcelona, the Consorci Sanitari Integral and the Servei Català de la Salut of the Generalitat de Catalunya. 